\title{
Editorial
}

\section{European Congress of Methodology}

\author{
Mirjam Moerbeek and Rens van de Schoot
}

Department of Methods and Statistics, Utrecht University, The Netherlands

From July 23 to 25, 2014, the VI European Congress of Methodology (http://eam2014.fss.uu.n1/) was held in Utrecht, the Netherlands. The European Congress of Methodology is organized biennially under the supervision of the European Association of Methodology (EAM; http://eamonline.org/), a society established in 2004, which brings together a large number of researchers from all over the world. The Congress provides a forum for specialists in scientific research methodology from all over Europe and beyond to meet and discuss the latest advances in the field of scientific methodology. The 2014 conference was organized by the Department of Methodology and Statistics at Utrecht University and was sponsored by Hogrefe Publishing, the city of Utrecht, the faculty of Social and Behavioural Sciences, and the Department of Methodology and Statistics.

We were happy to welcome 365 participants, not only come from Europe but also from countries as far away as Australia and Indonesia. Oral presentations were scheduled in 63 organized sessions, which were coordinated by experts in the respective field. Topics ranged from Bayesian statistics to survey methodology, and from statistical power analysis to analysis and reporting of interviews. The sessions were informal, with lots of room for discussion. Our social activities were a welcome reception and dinner in the beautiful medieval church and cloister garden of University Hall in the city center of Utrecht, where Utrecht University was founded in 1636.

Highlights of the conference were the award presentations for the best paper in Methodology to Ken Kelley (Kelley \& Cheng, 2012) and for best presentation by a student to Michèle Nuijten (2014), along with the election of Jacques Hagenaars as an honorary member of the European Association of Methodology.

Our invited presenters were Jacques Hagenaars (Tilburg University, The Netherlands), Tamas Rudas (Eötvös Loránd University, Budapest, Hungary), and Alicia O'Cathain (University of Sheffield, UK). The special issue of Methodology that you are reading now contains contributions from our three invited speakers that are based on their presentations.

Jacques Hagenaars' (2015) contribution is on methodological issues in categorical data analysis. Several ways of viewing categorical data, such as rating scales, are discussed. Hagenaars argues that rating scales (Likert items) might perhaps better be treated as strictly categorical, using latent class outcomes.

Tamas Rudas (2015) deals with the issue of measuring effects (causal or evidential) in the context of categorical data. Among other things, Rudas shows that measures of association do not appear to be automatically well suited to measuring effects and their differences, which he illustrates with Simpson's paradox. Rudas concludes that Simpson's paradox may only be avoided by using the cross sum ratio (CSR), but that the CSR is only appropriate under very specific conditions. Because of these very specific conditions, the paradox cannot be avoided in many decision-making situations.

Alicia O'Cathain's invited presentation is extended in Boeije, Drabble, and O'Cathain (2015), where the authors address the methodological challenges that accompany the use of a combination of research methods to evaluate complex interventions, called mixed methods intervention evaluation (MMIE). An example of such an MMIE is the combination of an RCT and qualitative research. The authors discuss how to choose appropriate designs for MMIEs, determining realistic expectations of both components and assigning adequate resources to each.

We hope to meet you all during the next conference, which is due to be held July 27-29, 2016, in La Palma de Mallorca.

\section{References}

Boeije, H., Drabble, S., \& O'Cathain, A. (2015). Methodological challenges of mixed methods intervention evaluations. Methodology, 11, 119-125. doi: 10.1027/1614-2241/a000101 
Hagenaars, J. A. (2015). Methodological issues in categorical data analysis: Categorization, linearity, and response effects. Methodology, 11, 126-141. doi: 10.1027/1614-2241/a000102

Kelley, K., \& Cheng, Y. (2012). Estimation and confidence interval formation for reliability coefficients of homogeneous measurement instruments. Methodology, 8, 39-50. doi: 10.1027/1614-2241/a000036

Nuijten, M. (2014, July). The replication paradox: Multiple studies with low power can increase bias. Paper presented at the VI European Congress of Methodology, Utrecht, The Netherlands.

Rudas, T. (2015). Effects and interactions. Methodology, 11, 142-149. doi: 10.1027/1614-2241/a000103
Rens van de Schoot

Department of Methods and Statistics

Utrecht University

P.O. Box 80.140

3508 TC Utrecht

The Netherlands

Tel. +31 30 253-4594

E-mail a.g.j.vandeschoot@uu.nl

Mirjam Moerbeek

Department of Methods and Statistics

Utrecht University

P.O. Box 80.140

3508 TC Utrecht

The Netherlands

Tel. +31 30 253-1450

E-mail m.moerbeek@uu.nl 